\title{
Analysis and numerical solution of epidemic models by using nonstandard finite difference scheme
}

\author{
Muhammad Farman ${ }^{1 *}$, Aqeel Ahmad ${ }^{1}$, Muhammad Umer Saleem ${ }^{2}$ and \\ Muhammad Ozair Ahmad ${ }^{1}$ \\ 1. Department of Mathematics and Statistics, University of Lahore-Pakistan \\ 2. Division of Science and Technology, University of Education, Lahore-Pakistan \\ *Corresponding author's email: farmanlink@ gmail.com \\ Citation \\ Muhammad Farman, Aqeel Ahmad, Muhammad Umer Saleem and Muhammad Ozair Ahmad. Analysis and \\ numerical solution of epidemic models by using nonstandard finite difference scheme. Pure and Applied Biology. \\ Vol. 9, Issue 1, pp674-682. http://dx.doi.org/10.19045/bspab.2020.90073
}

Received: 13/06/2019 Revised: 16/12/2019

Accepted: $17 / 12 / 2019$

Online First: 26/12/2019

\section{Abstract}

In this work, nonlinear epidemic models for measles, dengue fever, smoking, and SVIR are considered in order to understand the outbreaks of this epidemic disease. Developed unconditionally convergent nonstandard finite difference scheme by applying Mickens approach with $\emptyset(h)=h+O\left(h^{2}\right)$ as this will provide a feasible solution to overcome its disease risk as these are the main requirement for the dynamical systems in real-life solution. This method proved to be a very efficient technique for solving epidemic models to control infection diseases. Numerical simulations are carried out to support the analytic results.

Keywords: Epidemic model; Non-standard finite difference scheme; Qualitative analysis;

Stability analysis

\section{Introduction}

In the human population, epidemiology studies play an important role to understand the disease in the human population. Often the work of mathematical epidemiology consists of model building, estimation of parameters and investigation of the sensitivity of models to change in the parameters and numerical simulations. Epidemiologists use mathematical models to understand previous outbreaks of diseases and to better understand the dynamics of how infections spread through populations [1]. The research of this kind helps to understand the ratio of disease spread in the population and to control their parameters $[2,3]$. These types of diseased models are often called infectious diseases (i.e. the disease which transferred from one person to another person). Measles, rubella, chickenpox, mumps, aids and gonorrhea syphilis are examples of infectious disease $[4,5]$.

The Nonstandard Finite Difference (NSFD) schemes preserve the main properties of the differential counterparts, such as positivity, monotonicity, periodicity, stability, and some other invariant including energy and geometrical shapes. It should be emphasized that NSFD schemes can preserve all properties of continuous models for any discretization parameters. The discrete 
models with these properties are called dynamically consistent [6-9].

\section{SIR Model}

Kermack and McKendrick are those persons who are the inventor of the disease models and they played an important role in Mathematical epidemiology. In the purposed model, the population is divided into three groups which denoted by S (susceptible), I (infected) and R (recovered). The class $\mathrm{S}$ of susceptible is increased by birth at a rate $\pi N$ where both rate $\pi$ and death rate $\mu$ are the same in [7], so susceptible is increased by $\mu N$. It is decreased by infection following contacts with infected individuals I at rate $\alpha$. This class is decreased by recovery from infection at a rate $\gamma$ and dimensioned by natural death rate $\mu$. This generates a class $\mathrm{R}$ of individuals who have complete protection against disease and can be seen in (Figure 1).

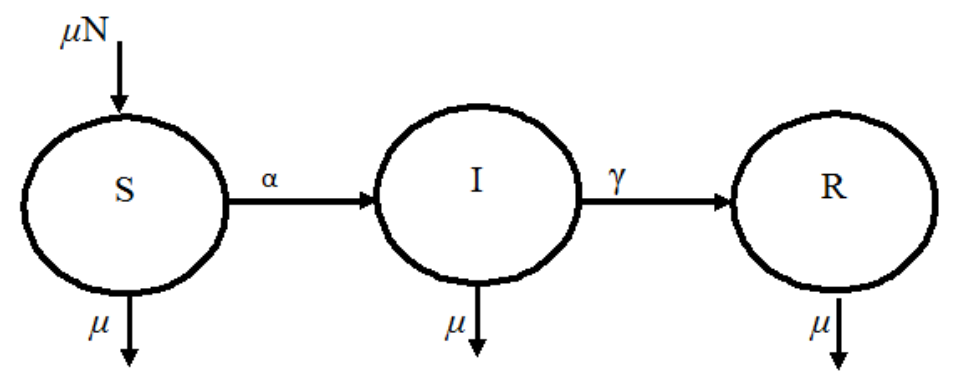

Figure 1. Flow chart of SIR model

Followings are the equations of the model

$\frac{d S}{d t}=\mu N-\alpha S I-\mu S$

$\frac{d I}{d t}=\alpha S I-(\gamma+\mu) I$

$\frac{d R}{d t}=\gamma I-\mu R$

Where $\mu, \alpha$ and $\gamma$ represents the per capita removal rate, transitivity rate ant per capita recovery rate respectively and $N=S+I+$
$R$, with initial conditions $S(0)=$ $990, I(0)=10, R(0)=0$

Qualitative analysis of the model

By simplifying the above equations we get disease-free equilibrium denoted by $E_{0}$ i.e $E_{0}=(N, 0,0)$ and the endemic equilibrium point denoted by $E_{1}$ i.e.

$E_{1}=\left(\frac{(\gamma+\mu)}{\alpha}, \frac{\alpha \mu N-\mu(\gamma+\mu)}{\alpha(\gamma+\mu)}, \frac{\alpha \gamma \mu N-\mu \gamma(\gamma+\mu)}{\alpha \mu(\gamma+\mu)}\right)$ and the reproductive number is $R_{0}=\frac{\alpha N}{(\gamma+\mu)}>1$

\section{Nonstandard finite difference (NSFD) scheme}

In recent years, NSFD discrete models have been constructed and or tested for a wide range of nonlinear dynamical systems. In this section, we design the NSFD scheme that replicates the dynamics of a continuous model (1-3). Let $Y_{k}=\left(S_{k}, I_{k}, R_{k}\right)^{t}$ denoted the approximation of $X\left(t_{k}\right)$ where $t_{k}=$ $k \Delta t$, with $k \epsilon N, h=\Delta t>0$ be a step size then:

$$
\begin{aligned}
& \frac{S^{k+1}-S^{k}}{\varnothing}=\mu N-\alpha S^{k+1} I^{k}-\mu S^{k+1} \\
& \frac{I^{k+1}-I^{k}}{\varnothing}=\alpha S^{k+1} I^{k}-(\gamma+\mu) I^{k+1}
\end{aligned}
$$


$\frac{R^{k+1}-R^{k}}{\emptyset}=\gamma I^{k+1}-\mu R^{k+1}$

$S^{k+1}=\frac{\mu N \emptyset+S^{k}}{1+\emptyset \alpha I^{k}+\emptyset \mu}$

$I^{k+1}=\frac{\left(\alpha \emptyset S^{k+1} I^{k}+I^{k}\right)}{1+\emptyset(\gamma+\mu)}$

$R^{k+1}=\frac{\gamma \emptyset I^{k+1}+R^{k}}{1+\emptyset \mu}$

Which is the purposed NSFD scheme for the given model, where

$\emptyset=\emptyset(h)=\frac{1-e^{-(\gamma+\mu) h}}{(\gamma+\mu)}$

Numerical simulations

The mathematical analysis of the epidemic measles SIR model with non-linear incidence has been presented. To observe the effects of the parameters using in this dynamics measles SIR model (1-3), conclude several numerical simulations with parameters values $\quad \alpha=0.003, \mu=0.05, \gamma=1, N=$ 1000 are given in [7]. In (Figure 2) shows that the model presented gradually approaches the steady states for different values of h. It is clearly shows that infected individuals at the latent period are diagnosed and treated, the number of susceptible individuals decreases significantly, the infected decreases steadily while the recovered increased steadily.

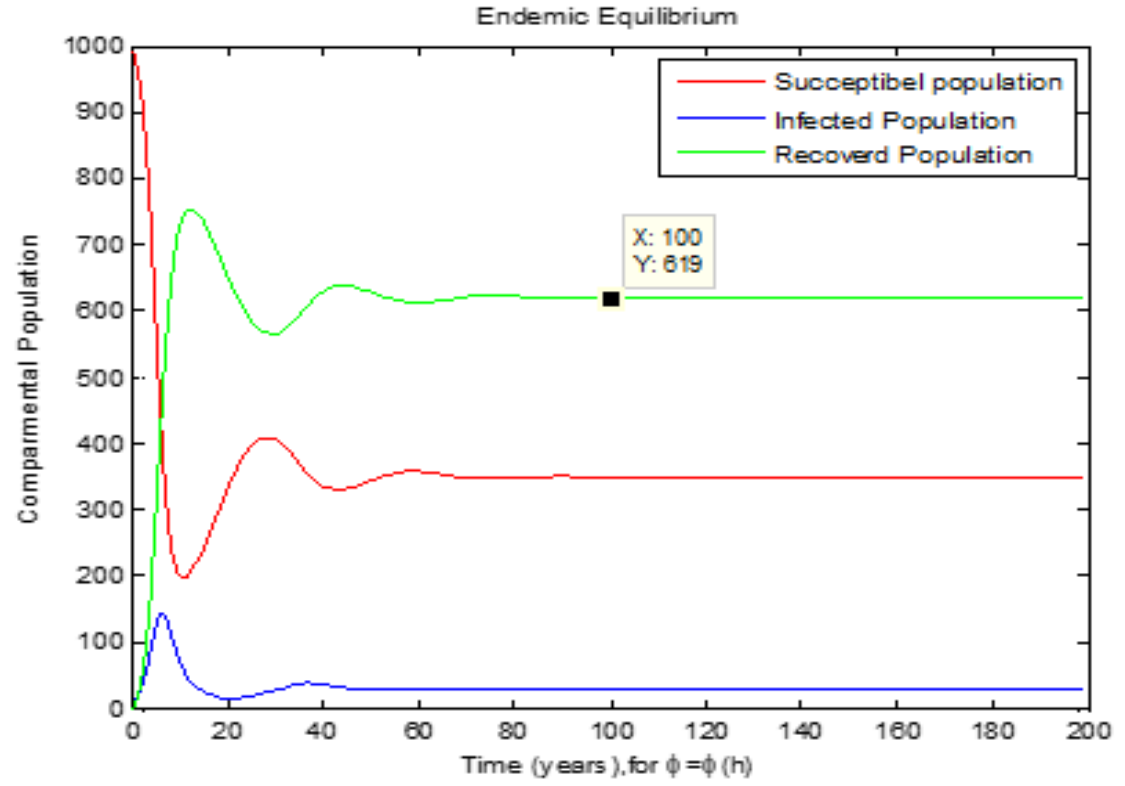

Figure 2. Numerical solutions for susceptible, infected individual, recovered population in a time $t$ for $\emptyset=\emptyset(h)$, where $h=10$

\section{Dengue model}

Esteva and Vargas [11] settled this model with the assumption that if a person gets the immunity against the disease, then the individuals will not be reinserted by this disease. In this model host population assuming constantly that means natural death rate and birth rate are equal. The differential equation of the dengue model as follows.

$$
\begin{aligned}
\frac{d S_{h}}{d t} & =A-\frac{\beta_{h} b}{N_{h}} S_{h} I_{v}-\mu_{h} S_{h} \\
\frac{d I_{h}}{d t} & =\frac{\beta_{h} b}{N_{h}} S_{h} I_{v}-\left(\mu_{h}+\gamma\right) I_{h} \\
\frac{d R_{h}}{d t} & =\gamma I_{h}-\mu_{h} R_{h} \\
\frac{d S_{v}}{d t} & =B-\frac{\beta_{v} b}{N_{h}} S_{v} I_{h}-\mu_{v} S_{v} \\
\frac{d I_{v}}{d t} & =\frac{\beta_{v} b}{N_{h}} S_{v} I_{h}-\mu_{v} I_{v}
\end{aligned}
$$


Where

$S_{h}, I_{h}, R_{h}, N_{v}, S_{v}, I_{v}, \mu_{v}, \beta_{h}, \beta_{v}, \gamma, b, A, B$ are host population of susceptible individuals, host population of infected individuals, host population of permanent immune individuals, vector population, vector population of susceptible individuals, vector population of infected individuals, death rate of vector population, transmission probability from vector to host, transmission probability from host to vector, rate of recovery from host population, biting rate of vector, recruitment rate of host population, Recruitment rate of host population respectively.

\section{Nonstandard finite difference (NSFD) scheme of the model}

In this section, we design the NSFD [8-10] scheme that replicates the dynamics of a continuous model (1)-(5). Let $Y_{k}=$ $\left(\left(S_{h}\right)_{k},\left(I_{h}\right)_{k},\left(R_{h}\right)_{k},\left(S_{v}\right)_{k},\left(I_{v}\right)_{k}\right)^{t}$ denoted the approximation of $X\left(t_{k}\right)$ where $t_{k}=$ $k \Delta t$, with $k \in N, h=\Delta t>0$ be a step size then;

$\frac{S_{h}^{k+1}-S_{h}^{k}}{\emptyset}=A-\frac{\beta_{h}}{N_{h}} b S_{h}^{k+1} I_{v}^{k}-\mu_{h} S_{h}^{k+1}$

$\frac{I_{h}^{k+1}-I_{h}^{k}}{\emptyset}=\frac{\beta_{h}}{N_{h}} b S_{h}^{k+1} I_{v}^{k}-\left(\gamma+\mu_{h}\right) I_{h}^{k+1}$

$\frac{R_{h}^{k+1}-R_{h}^{k}}{\varnothing}=\gamma I_{h}^{k+1}-\mu_{h} R_{h}^{k+1}$

$\frac{S_{v}^{k+1}-S_{v}^{k}}{\varnothing}=B-\frac{\beta_{v}}{N_{h}} b S_{v}^{k+1} I_{h}^{k}-\mu_{v} S_{v}^{k+1}$

$\frac{I_{v}^{k+1}-I_{v}^{k}}{\emptyset}=\frac{\beta_{v}}{N_{h}} b S_{v}^{k+1} I_{h}^{k}-\mu_{v} I_{v}^{k+1}$

$S_{h}^{k+1}=\frac{A \emptyset+S_{h}^{k}}{1+\frac{\beta_{h}}{N_{h}} b \emptyset I_{v}^{k}+\emptyset \mu_{h}}$

$I_{h}^{k+1}=\frac{\frac{\beta_{h}}{N_{h}} b \emptyset S_{h}^{k+1} I_{v}^{k}+I_{v}^{k}}{1+\emptyset\left(\gamma+\mu_{h}\right)}$

$R_{h}^{k+1}=\frac{\gamma \emptyset I_{h}^{k+1}+R_{h}^{k}}{1+\emptyset \mu_{h}}$

$S_{v}^{k+1}=\frac{B \emptyset}{1+\frac{\beta \beta_{h}}{N_{h}} b \emptyset I_{h}^{k}+\emptyset \mu_{v}}$

$I_{v}^{k+1}=\frac{\frac{\beta v}{N_{h}} b \emptyset S_{v}^{k+1} I_{h}^{k}+I_{v}^{k+1}}{1+\emptyset \mu_{v}}$

Which is the purposed NSFD scheme for the given model, where $\varnothing=\varnothing(h)=\frac{1-e^{-\left(\gamma+\mu_{h}\right) h}}{\left(\gamma+\mu_{h}\right)}$

The discrete method (13-17) is certainly defined by using Mickens's rules.

Numerical simulation

The mathematical analysis of the vectorborne disease dengue fever model with nonlinear incidence has been presented. To observe the effects of the parameters using in this dynamics Dengue epidemic model (712), conclude several numerical simulations with parameters values $A=0.457, B=$ $400, \beta_{h}=0.75, b=0.5, \mu_{h}=$ $0.0000457, \gamma=0.1428, N_{h}=1000, \beta_{v}=$ $1, \mu_{v}=0.25$ are given in [11]. In (Figure3) shows the convergence solution using the NSFD scheme at $h=1$ for the true equilibrium point. It can be easily seen from (Figure3) which shows fast convergence for each compartment. It clearly shows that infected individuals at the latent period are diagnosed and treated, the number of susceptible individuals decreases significantly, the infected decreases steadily.

\section{Smoking Model}

Smoking model is divided into five subcompartment like potential smokers $P(t)$, occasional smokers $L(t)$, heavy smoker's $S(t)$ temporary quitters $Q(t)$ and smokers who quit permanently $R(t)$ specified $\operatorname{by} T(t)=P(t)+L(t)+S(t)+Q(t)+$ $R(t)$. The proposed smoking [12] in the form of the system of the nonlinear differential equation is given as:

$$
\begin{aligned}
& \frac{d P}{d t}=a(1-P)-b P S \\
& \frac{d L}{d t}=-a L+b P S-c L S \\
& \frac{d S}{d t}=-(a+d) S+c L S+f Q \\
& \frac{d Q}{d t}=-(a+f) Q+d(1-e) S \\
& \frac{d R}{d t}=-a R+e d S
\end{aligned}
$$

Here $b$ indicates the contact rate between potential smokers and smokers who smoke occasionally, $c$ represents the contact rate 
between temporary quitters and smokers who smoke occasionally, $d$ represents the rate of giving up smoking, $(1-e)$ stances for the fraction of smokers who temporarily give up smoking at a rate $d, f$ indicate the contact rate between smokers and temporary quitters who return back to smoking, $a$ denotes the rate of natural death, $e$ denotes the remaining fraction of smokers who give up smoking permanently (at a rate $d$ ).

Nonstandard finite difference (NSFD) scheme of the model

Let $Y_{k}=\left(P_{k}, L_{k}, S_{k}, Q_{k}, R_{k}\right)^{T}$ denoted an approximation of $X\left(t_{k}\right)$ where $t_{k}=k \Delta t$ with $k \in N, h=\Delta t>0$ be a step size, then

$\frac{P^{k+1}-P^{k}}{\emptyset}=a-a P^{k+1}-b P^{k+1} S^{k}$

$\frac{L^{k+1}-L^{k}}{\varnothing}=-a L^{k+1}+b P^{k+1} S^{k}-c L^{k+1} S^{k}$

$\frac{S^{k+1}-S^{k}}{\varnothing}=-(a+d) S^{k+1}+c L^{k+1} S^{k}+f Q^{k}$

$\frac{Q^{k+1}-Q^{k}}{\emptyset}=-(a+f) Q^{k+1}+d(1-e) S^{k+1}$

$\frac{R^{k+1}-R^{k}}{\emptyset}=-a R^{k+1}+e d S^{k+1}$

$P^{k+1}=\frac{a \emptyset+P^{k}}{1+a \emptyset+b \emptyset S^{k}}$

$L^{k+1}=\frac{b \emptyset P^{k+1} S^{k}+L^{k}}{1+a \emptyset+c \emptyset S^{k}}$

$S^{k+1}=\frac{\emptyset c L^{k+1} S^{k}+\emptyset f Q^{k}+S^{k}}{1+(a+d) \emptyset}$

$$
\begin{aligned}
Q^{k+1} & =\frac{\emptyset d(1-e) S^{k+1}+Q^{k}}{1+\emptyset(a+f)} \\
R^{k+1} & =\frac{R^{k}+\emptyset e d S^{k+1}}{1+a \emptyset}
\end{aligned}
$$

Which is the proposed NSFD Scheme for the given model, where

$\emptyset=\emptyset(h)=\frac{1-e^{-d(1-e) h}}{d(1-e)}$

\section{Numerical simulation}

The technique creates a better impact to control the smoking, it reduces the infected rate and increases the potential smokers during disease Free State. In (Figure 4) by decreasing the value of $h$ potential smokers $(P)$ increases with time.In (Figure 5) by decreasing the value of $h$ occasional smokers $(L)$ decreases rapidly with time. In (Figure 6) by decreasing the value of $h$ heavy smokers $(S)$ decreases rapidly with time. In (Figure 7), by decreasing the value of $h$ temporary quitters $(Q)$ increases initially, but after some time decreases rapidly with time. In (Figure 8 ) by decreasing the value of $h$ permanent quitters $(R)$ increases initially, but after some time decreases rapidly with time. It can be easily seen that by reducing the step size the system (18-22) converge rapidly to the steady-state point [13].

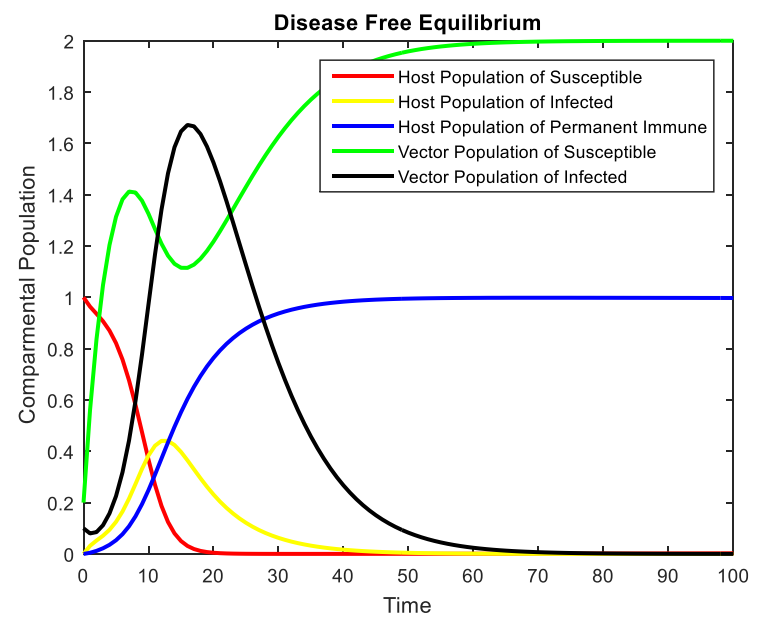

Figure 3. Host Population of Susceptible individual, Host Population of Infected Individual, Host Population of Permanent Immune individual, Vector Population of Susceptible, Vector Population of Infected 


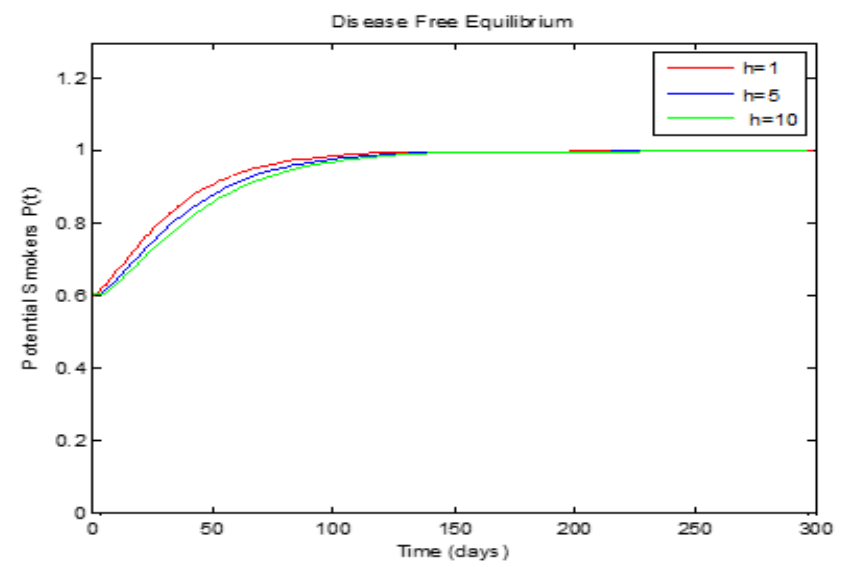

Figure 4. Numerical solutions for potential smokers in a time $t$ with step size $h=1, h=5$ and $h=\mathbf{1 0}$ for disease free equilibrium points

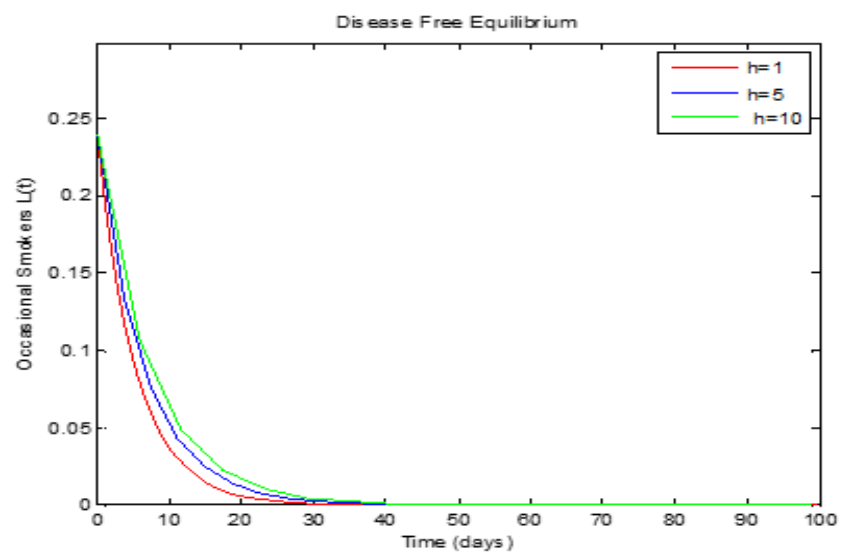

Figure 5. Numerical solutions for occasional smokers in a time $t$ with step size $h=1, h=5$ and $h=10$ for disease free equilibrium points

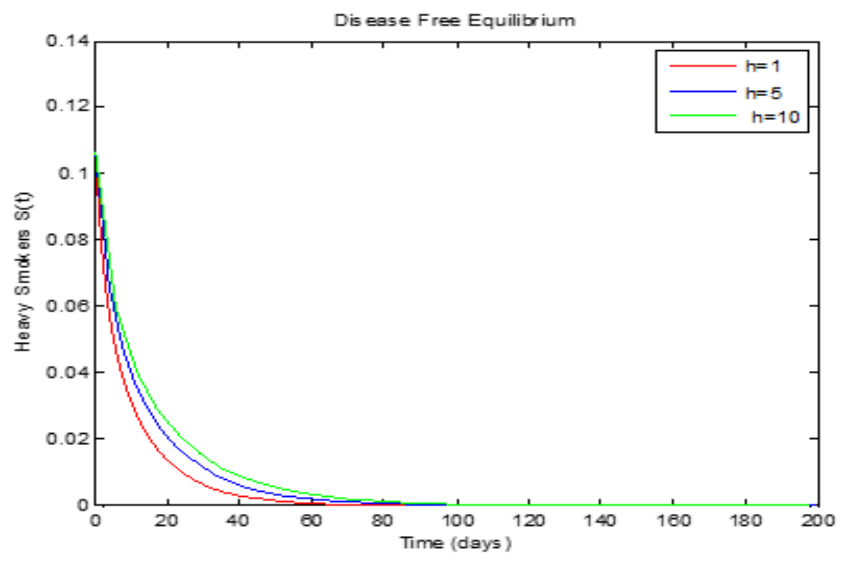

Figure 6. Numerical solutions for heavy smokers in a time $t$ with step size $h=1, h=5$ and $h=10$ for disease free equilibrium points 


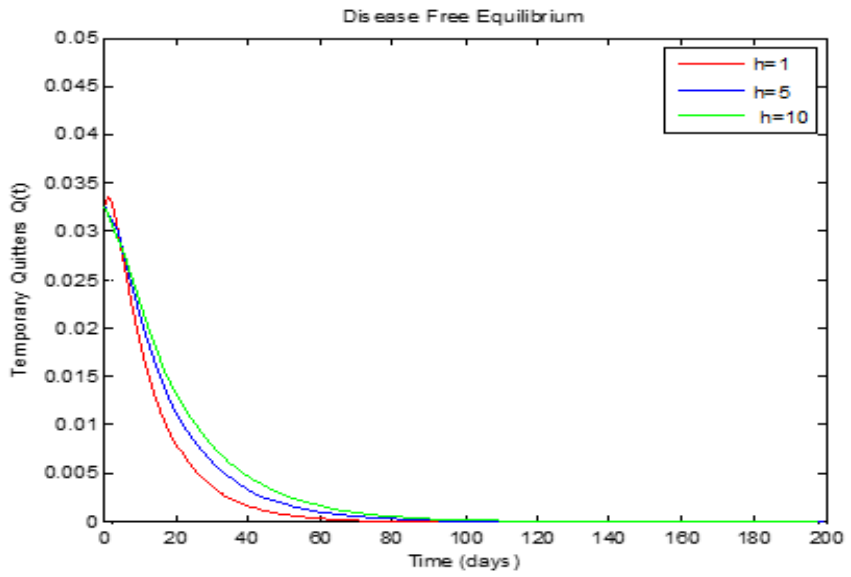

Figure 7. Numerical solutions for temporary quitters in a time $t$ with step size $h=1, h=5$ and $h=10$ for disease free equilibrium points

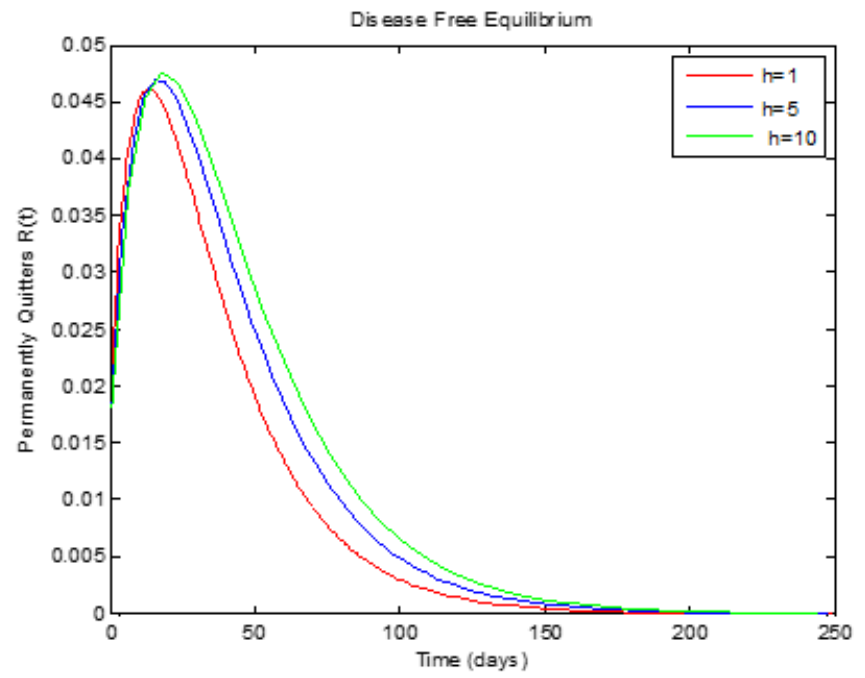

Figure 8. Numerical solutions for permanently quitters in a time $t$ with step size $h=1, h=$ 5 and $h=10$ for disease-free equilibrium points

SVIR model

$$
\begin{aligned}
& \frac{d S}{d t}=\mu-\mu S-\beta S I-\alpha S \\
& \frac{d V}{d t}=\alpha S-\beta_{1} V I-\gamma_{1} V-\mu V \\
& \frac{d S}{d t}=\beta S I+\beta_{1} V I-\gamma I-\mu I \\
& \frac{d S}{d t}=\gamma_{1} V+\gamma I-\mu R
\end{aligned}
$$

Here $S(t), I(t)$ and $R(t)$ denote the densities of susceptible, infected and recovered individuals, respectively. $V(t)$ denotes the density of vaccines who have begun the vaccination process. In the above model, all parameters are positive and have the following features: $\mu$ is the recruitment rate and natural death rate of the population; $\beta$ 
denotes the transmission coefficient between compartments $S$ and $I ; \alpha$ is the rate at which susceptible individuals are moved into the vaccination process; $\beta_{1}$ denotes the disease transmission rate when the vaccines contact with infected individuals before obtaining immunity; $\gamma_{1}$ is the average rate for the vaccines move into recovered population; $\gamma$ is the recovery rate of infected individuals

\section{Qualitative analysis}

For the equilibrium points, we take $\frac{d s}{d t}=$ $0, \frac{d V}{d t}=0, \frac{d I}{d t}=0, \frac{d R}{d t}=0$ then we get

$0=\mu-\mu S-\beta S I-\alpha S$

$0=\alpha S-\beta_{1} V I-\gamma_{1} V-\mu V$

$0=\beta S I+\beta_{1} V I-\gamma I-\mu I$

$0=\gamma_{1} V+\gamma I-\mu R$

By solving the above model we get [14].

$$
S_{E}(S, I, V, R)=S_{E}\left(\frac{\mu}{\alpha+\mu}, 0, \frac{\alpha \mu}{(\alpha+\mu)\left(\mu+\gamma_{1}\right)}, \frac{\alpha \gamma_{1}}{(\alpha+\mu)\left(\mu+\gamma_{1}\right)}\right)
$$

Nonstandard finite difference (NSFD) scheme of the model

Followings are the scheme

$S^{k+1}=\frac{h \mu+S^{k}}{1+h \mu+h \beta I^{k}+h \alpha}$

$V^{k+1}=\frac{\left(h \alpha S^{k+1}+V^{k}\right)}{1+h \beta_{1} I^{k}+h \gamma_{1}+h \mu}$

$I^{k+1}=\frac{h \beta S^{k+1} I^{k}+h \beta V^{k+1} I^{k}}{1+h \gamma+h \mu}$

$R^{k+1}=\frac{h \gamma_{1} V^{k+1}+R^{k}}{1+h \mu}$

The mathematical analysis of the epidemic SVIR (Susceptible Vaccination Infected
Recovered) model with non-linear incidence has been presented. To observe the effects of the parameters using in this dynamics SVIR model, conclude several numerical simulations with the given parameter values. In (Figure 9) shows that the model presented gradually approaches the steady states and infected individuals at the latent period are diagnosed and treated after vaccination, the number of susceptible individual's decreases significantly; the infected decreases steadily while the recovered increased rapidly.

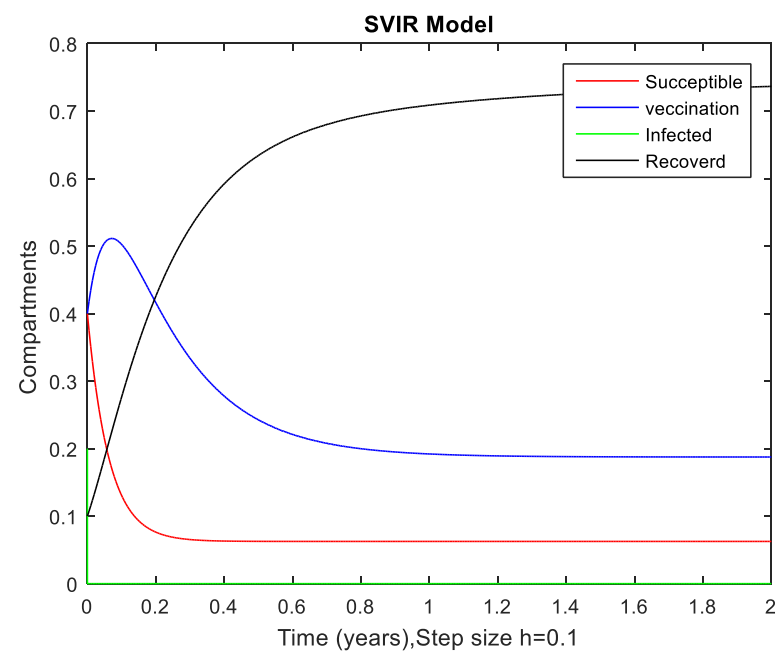

Figure 9. Numerical solutions for susceptible, vaccination, infected individual and recovered population in a time $t$ for $\emptyset=\emptyset(h)$ 


\section{Conclusion}

Numerical simulations are performed and all the analytical results are verified numerically by using the Non-Standard Finite Difference Scheme to reduce the infected rate in different epidemic models. This leads to the practical implications for the control of measles infections, dengue fever, smoking and SVIR by these models. It can easily be seen from the developed scheme and their graphical representations that NSFD preserves all the mandatory properties of continuous models like positivity, boundednes etc. for epidemic models, while all other numerical techniques fail to maintain the properties of continuous models for the larger step sizes. NSFD Scheme also always converges to their steady-state points while other numerical techniques like Adomian Decomposition Method (ADM), Differential Transform Method (DTM), Runge Kutta Method and Euler's Methods etc. fails to do so. This can help to control the spreading of infectious diseases in the community.

\section{Authors' contributions}

Conceived and designed the experiments: $\mathrm{M}$ Farman \& A Ahmad, Performed the experiments: MU Saleem \& M Farman, Analyzed the data: A Ahmad \& MO Ahmad, Contributed materials/ analysis/ tools: MU Saleem \& MO Ahmad, Wrote the paper: M Farman \& A Ahmad.

\section{References}

1. Abubakar S, Akinwande NI \& Abdulrahman (2012). A Mathematical Model of Yellow Fever Epidemics, Afrika Mathematika 6: 5658.

2. Grenfell $\mathrm{T}$ (1992). Chance and chaos in measles dynamics. J R Stat Soc B 54: 383398.

3. Panum PL (1940). Observations Model during the epidemic of Measles on the Faroe
Islands in the year 1846, Delta Omega Society, Cleveland.

4. WHO (2010). Vaccine-preventable diseases: monitoring system. Geneva: World Health Organization.

5. Ministry of Health, Manatu Hauora (1998). Predicting and Preventing Measles Epidemic in New Zealand: Application of Mathematical Model.

6. Anguelov R, Dumont Y, Lubuma JMs \& Shillor M (2014). Dynamically consistent nonstandard finite differences schemes for epidemiological models. J of Comp and Appl Maths 225: 161-182

7. Anguelov R \& Lubuma JMS (2001). Contribution to the mathematics of the nonstandard finite difference method and applications, Numer. Methods Partial Differ. Equ 17: 518-543.

8. Lubuma JMS \& Patidar K (2007). Nonstandard methods for singularity perturbed problems possessing oscillatory/ layer solutions, Appl Maths Comp 187(2). 11471160 .

9. Roeger LW (2014). Exact difference schemes, in A. B. Gumel Mathematics of continuous and discrete Dynamics Systems. Contemp Maths 618: 147-161.

10. Mickens RE (1989). Exact solutions to a finite difference model of a nonlinear reactions advection equation: Implications for numerical analysis. Numer Meth for Partial Diff Equa 5: 313-325.

11. Hamed AL, Moustafa ElS, Juan J \& Nieto WS (2014). On fractional Order Dengue Epidemic Model. Mathem Prob in Engin, doi.org/10.1155,456537, 1-6.

12. Singh J, Kummar D, Qurashi M \& Baleanu D (2017). A new fractional model for giving up smoking dynamics. Adv in Diff Equa 88.

13. Aqeel A, Farman M, Faisal Y \& Ahmad M O (2018). Dynamical transmission and effect of smoking in society. Inter $J$ of $A d v$ and Appl Sci 5(2): 71-75.

14. Liu Y, Takeuchi \& Awarni S (2008). SVIR epidemic models with vaccination strategies. J Theoret Biol 253. 